\title{
Training-Induced Deactivation of the AT1 Receptor Pathway Drives Autonomic Control and Heart Remodeling During the Transition From the Pre- to Hypertensive Phase in Spontaneously Hypertensive Rats
}

\author{
Tassia Santos Rodrigues da Costa, BSc; Gustavo Santos Masson, PhD; \\ Rosangela Aparecida dos Santos Eichler, PhD; Juliane Cristina de Souza Silva, BSc; \\ Silvia Lacchini, PhD; Lisete Compagno Michelini, PhD
}

\begin{abstract}
Background: The effects of hypertension and exercise training $(T)$ on the sequential interplay between renin-angiotensin system (RAS), autonomic control and heart remodeling during the development of hypertension in spontaneously hypertensive rats (SHR), was evaluated.
\end{abstract}

Methods and Results: Time course changes of these parameters were recorded in 4-week-old SHR submitted to a T or sedentary (S) protocol. Wistar Kyoto rats served as controls. Hemodynamic recordings were obtained in conscious rats at experimental weeks $0,1,2,4$, and 8 . The left ventricle (LV) was collected to evaluate RAS gene and protein expression, cardiomyocytes' hypertrophy and collagen accumulation. Pre-hypertensive SHR exhibited augmented AT ${ }_{1}$ R gene expression; at 5 weeks, they presented with elevated pressure, increased LV angiotensinogen and ACE mRNA expression, followed by sympathoexcitation (from the $8^{\text {th }}$ week onwards). Marked AT 1 R protein content, myocytes's hypertrophy, collagen deposition and increased pressure variability were observed in 12-weekold sedentary SHR. In addition to attenuating all these effects, T activated Mas receptor expression augmented parasympathetic modulation of the heart, and delayed the onset and reduced the magnitude, but did not block the development of genetic hypertension.

Conclusions: The close temporal relationship between changes in the LV ACE-Ang II-AT R axis, autonomic control and cardiac remodeling at both the establishment of hypertension and during exercise training reveals the essential role played by the $A T_{1} R$ pathway in driving cardiac remodeling and autonomic modulation during the transition from the pre- to hypertensive phase.

Key Words: Collagen content; Exercise training; Juvenile spontaneously hypertensive rats; Myocytes' hypertrophy; Reninangiotensin system

$\mathbf{E}$ ssential hypertension is a multifactorial chronic disease involving complex interactions between oxidative stress, pro-inflammatory profile, autonomic and endothelial dysfunction, immune system hyperactivity and arterial remodeling. ${ }^{\mathbf{1} 2}$ These deleterious responses are important risk factors for cardiovascular morbidity and mortality. Although more prevalent in advanced ages, some pathophysiological abnormalities such as altered autonomic control, ${ }^{3,4}$ increased cardiac sympathetic neurotransmission, ${ }^{5}$ endothelial dysfunction, ${ }^{6}$ and vascular endoplasmic reticulum stress ${ }^{7}$ were already observed in young- to middle-aged hypertensive individuals. Experimental evidence suggests that angiotensin II (Ang II) and angiotensin-(1-7) [Ang-(1-7)], the main active hormones of the renin-angio- tensin system (RAS), are essential players in hypertensioninduced abnormalities. ${ }^{\mathbf{8}, 9}$

Among hypertension-induced end-organ lesions, hypertrophic cardiac remodeling is a well-known characteristic. This pathological process consists in concentric left ventricle (LV) hypertrophy, increased myocyte cross-sectional area, interstitial inflammation, extracellular collagen deposition, cardiac fibrosis, capillary rarefaction and apoptosis. ${ }^{10}$ Experimental studies have identified a close relationship between RAS expression, oxidative stress, local inflammation and cardiac remodeling in distinct experimental models of hypertension, ${ }^{11-13}$ as in diabetes. ${ }^{14}$ These studies confirmed clinical findings that $\mathrm{AT}_{1}$ receptor $\left(\mathrm{AT}_{1} \mathrm{R}\right)$ blockade reduced LV hypertrophy, highlighting the importance

Received January 30, 2020; revised manuscript received April 21, 2020; accepted April 30, 2020; J-STAGE Advance Publication released online June 10, 2020 Time for primary review: 29 days

Department of Physiology and Biophysics (T.S.R.daC., G.S.M., L.C.M.), Department of Pharmacology (R.A.dosS.E.), Department of Anatomy (J.C.deS.S., S.L.), Biomedical Sciences Institute, University of Sao Paulo, São Paulo, SP, Brazil

The first two authors contributed equally to the study (T.S.daR.C., G.S.M.).

Mailing address: Lisete Compagno Michelini, PhD, Department of Physiology and Biophysics, Institute of Biomedical Sciences, University of Sao Paulo, Av. Prof. Lineu Prestes 1524, 05508-000, São Paulo - SP - Brazil. E-mail: michelin@usp.br

ISSN-1346-9843 All rights are reserved to the Japanese Circulation Society. For permissions, please e-mail: cj@j-circ.or.jp 


\begin{tabular}{|c|c|c|c|}
\hline Gene & Sequence (5'-3') & $\begin{array}{l}\text { Length } \\
\text { (base pairs) }\end{array}$ & $\begin{array}{l}\text { Access number } \\
\text { (ncbi.nlm.nih.gov) }\end{array}$ \\
\hline \multirow[t]{2}{*}{ GAPDH } & S: GGG CAG CCC AGA ACA TCA T & 76 & NM 017008.4 \\
\hline & A: CCG TTC AGC TCT GGG ATG AC & & \\
\hline \multirow[t]{2}{*}{ Aogen } & S: CAC GGA CAG CAC CCT ATT TT & 100 & NM 134432.2 \\
\hline & A: TGT TGT CCA CCC AGA ACT CA & & \\
\hline \multirow[t]{2}{*}{ ACE } & S: GGG CGC ACG ACA CCA ACA TCA & 69 & NM 012544.1 \\
\hline & A: TCC TGG TTG ATC AGG GCC GCT T & & \\
\hline \multirow[t]{2}{*}{ ACE2 } & S: ACC CTT CTT ACA TCA GCC CTA CTG & 73 & NM 001012006.1 \\
\hline & A: TGT CCA AAA CCT ACC CCA CAT AT & & \\
\hline \multirow[t]{2}{*}{$A T_{1} R$} & S: CAC TTT CCT GGA TGT GCT GA & 140 & NM 030985.4 \\
\hline & A: CCC AGA AAG CCG TAG AAC AG & & \\
\hline \multirow[t]{2}{*}{ MasR } & S: TCC ACC AAG ACC TGC TAG GA & 208 & NM 012757.2 \\
\hline & A: TCT TGT GCT GGA CCA CTT CA & & \\
\hline
\end{tabular}

$S$ and $A$ indicate the sense and antisense sequences, respectively. Length is the amplicon size in base pairs; NM represents the access number from the ncbi.nlm.nih.gov site.
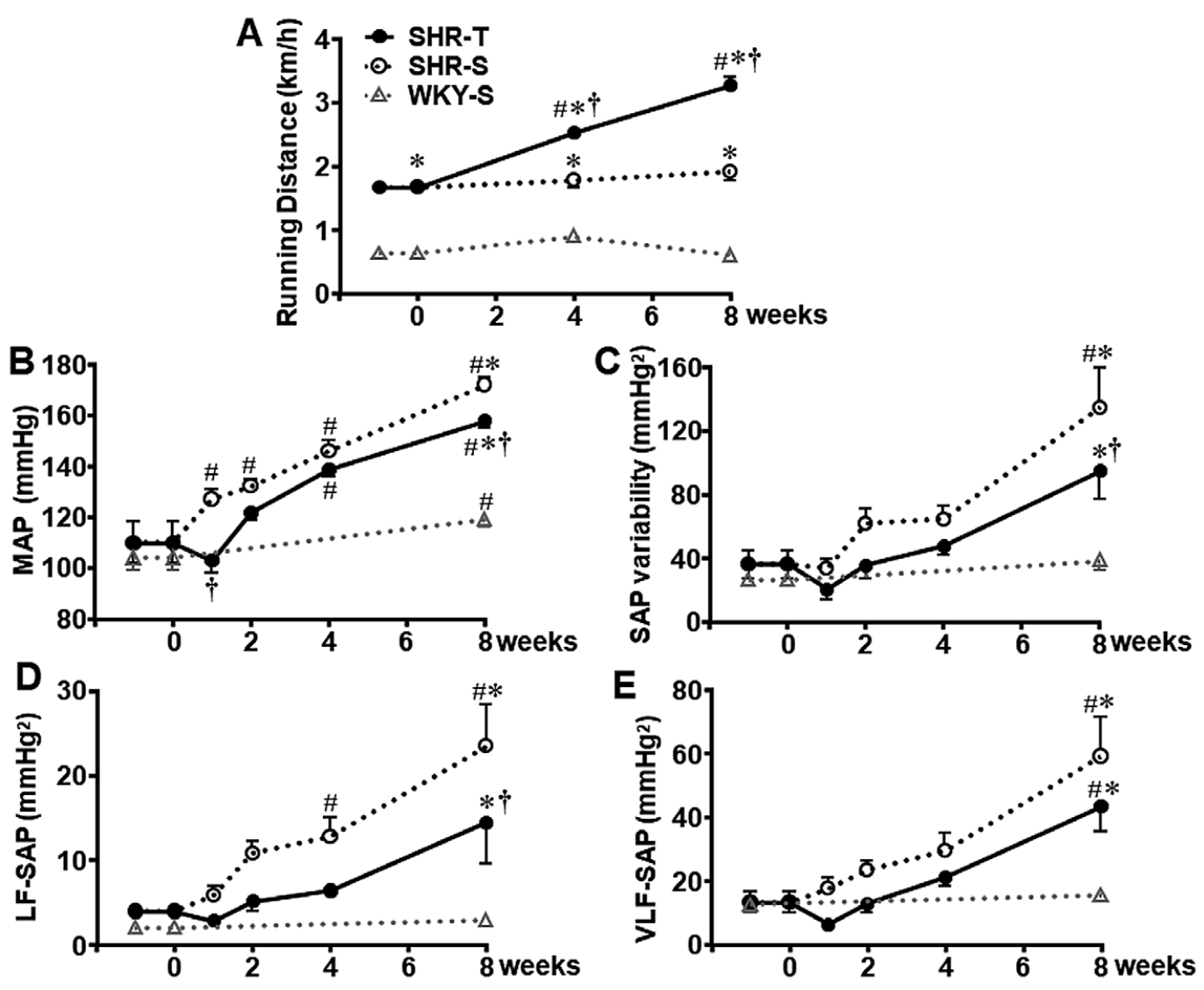

Figure 1. Hypertension- and training-induced changes on functional parameters during the development of hypertension in spontaneously hypertensive rats (SHR). Sequential changes on running distance (A), mean arterial pressure (MAP, B), systolic arterial pressure (SAP) variability (C) and its low frequency (LF-SAP, D) and very low frequency (VLF-SAP, E) components in SHR and normotensive controls (Wistar Kyoto (WKY)). MAP, SAP-variability and respective components were measured in 7-10/rats/time point/group. Training/Sedentary protocols started with 60 SHR and 25 WKY. Significance $(P<0.05)$ : \#vs. respective week 0; *Vs. age-matched WKY; † vs. age-matched SHR-sedentary (S). 

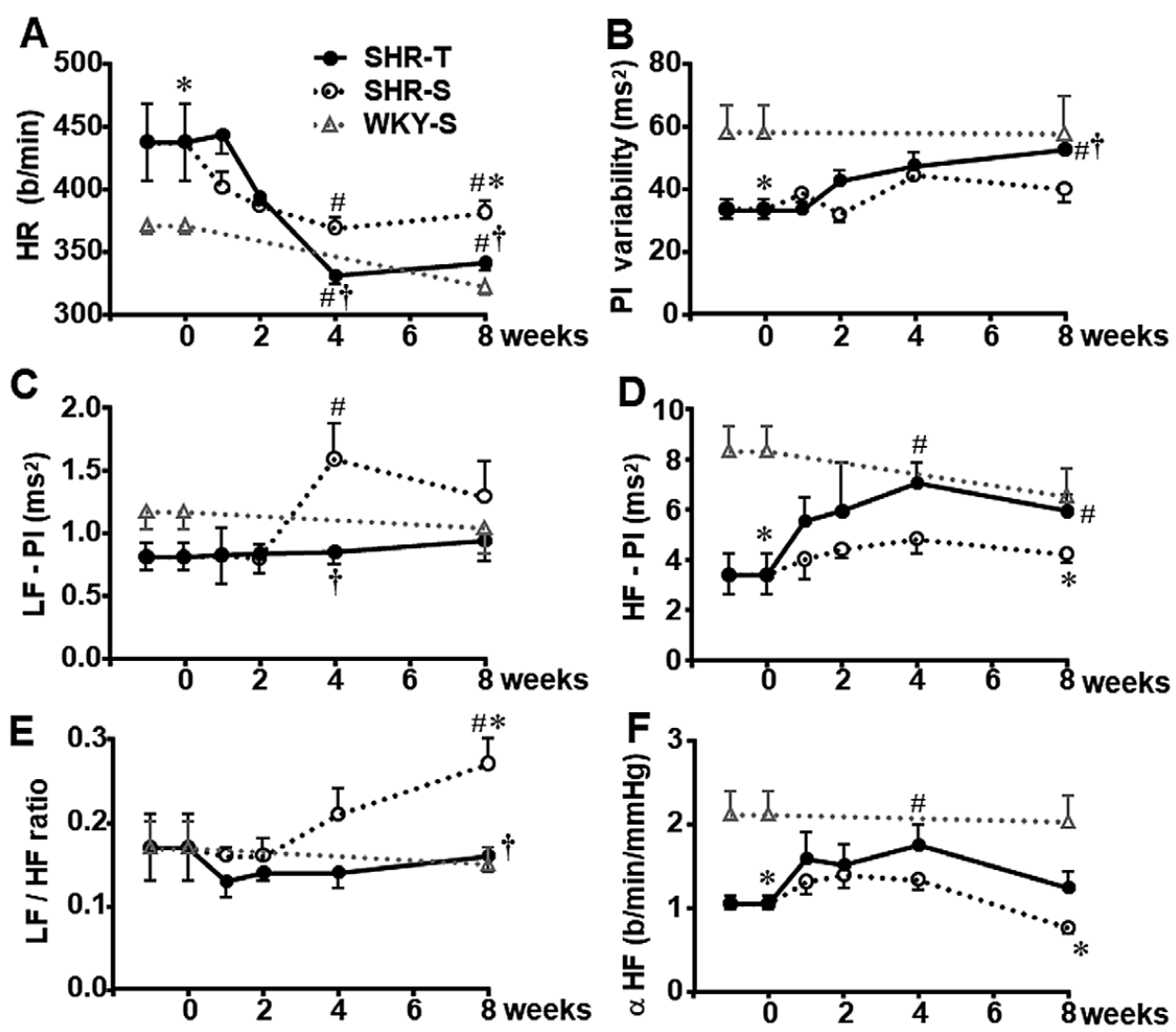

Figure 2. Hypertension- and training-induced changes on functional parameters during the development of hypertension in spontaneously hypertensive rats $(\mathrm{SHR})$. Sequential changes on heart rate $(\mathrm{HR}, \mathbf{A})$, pulse interval $(\mathrm{PI})$ variability $(\mathbf{B})$ and its low frequency (LF, C), high frequency (HF, D) components, LF/HF ratio, (E) and spontaneous baroreflex sensitivity $(a \mathrm{HF}, \mathbf{F})$ in SHR and normotensive controls (Wistar Kyoto (WKY)). SAP-variability and respective components were measured in 7-10/rats/time point/group. Significance $(P<0.05)$ : \#vs. respective week 0; *Vs. age-matched WKY; †vs. age-matched SHR-sedentary (S).

of the ACE-Ang II-AT 1 R axis. ${ }^{8}$ Other studies have shown that ACE2-Ang-(1-7)-Mas receptor (MasR) axis, the contraregulatory axis, also exhibits cardioprotective effects. ${ }^{15,16}$ Apart from pharmacological interventions, it was also shown that exercise training was highly effective in reducing the activity of the vasoconstrictor-trophic-pro-oxidativepro-inflammatory axis, while improving the contraregulatory RAS axis. ${ }^{\mathbf{1 3}, \mathbf{1 7 - 2 1}}$ All these studies, however, analyzed RAS expression/activity and the cardiac remodeling in sedentary and trained animals mainly in the chronic phase, not during the establishment of hypertension. A recent study investigated, in spontaneously hypertensive rats (SHR), the effects of exercise training starting at the pre-hypertensive phase on temporal changes of blood pressure levels, Ang II and Ang-(1-7) content and cardiac remodeling. ${ }^{22}$ However, it did not analyze the interplay between RAS, cardiac remodeling and autonomic control of the heart during the transition from pre- to hypertensive phase; this analysis is lacking.

In the present study, we investigated in young SHR, the effects of exercise training on the sequential changes of LV ACE-Ang II-AT 1 R and ACE2-Ang-(1-7)-MasR RAS axes, the associated responses of LV hypertrophy, myocyte crosssectional area and extracellular collagen deposition during the transition from the pre- to hypertensive phase. We also analyzed the temporal changes of resting arterial pressure (AP) and heart rate (HR), their variabilities and spectral components indicative of sympathetic and parasympathetic activity to heart and vessels. Wistar Kyoto rats (WKY) rats served as controls.

\section{Methods}

Young male SHR and WKY (21-22 days old) from the Animal Breeding Facility of the University of São Paulo were maintained in the Animal Facility of the Department of Physiology and Biophysics at a controlled room temperature $\left(-23^{\circ} \mathrm{C}\right)$, in a 12 -h dark-light cycle and had free access to standard chow and water. All surgical procedures and experimental protocols were in accordance with the National Institutes of Health, Guide for Care and Use of Laboratory Animals. The study was approved by the Institutional Committee of Animal Care and Use of Biomedical Sciences Institute, University of Sao Paulo $\left(\mathrm{n}^{0} 10\right.$, page 124, book 02).

After a 1-week adaptation period, the aerobic capacity was evaluated., $3,13,23$ SHRs were allocated to moderate exercise training $(\mathrm{T})$ or sedentary $(\mathrm{S})$ protocols for 8 weeks. At weeks $0,1,2,4$ and 8 (SHR) and weeks 0 and 8 (WKY), rats were chronically cannulated for hemodynamic record- 

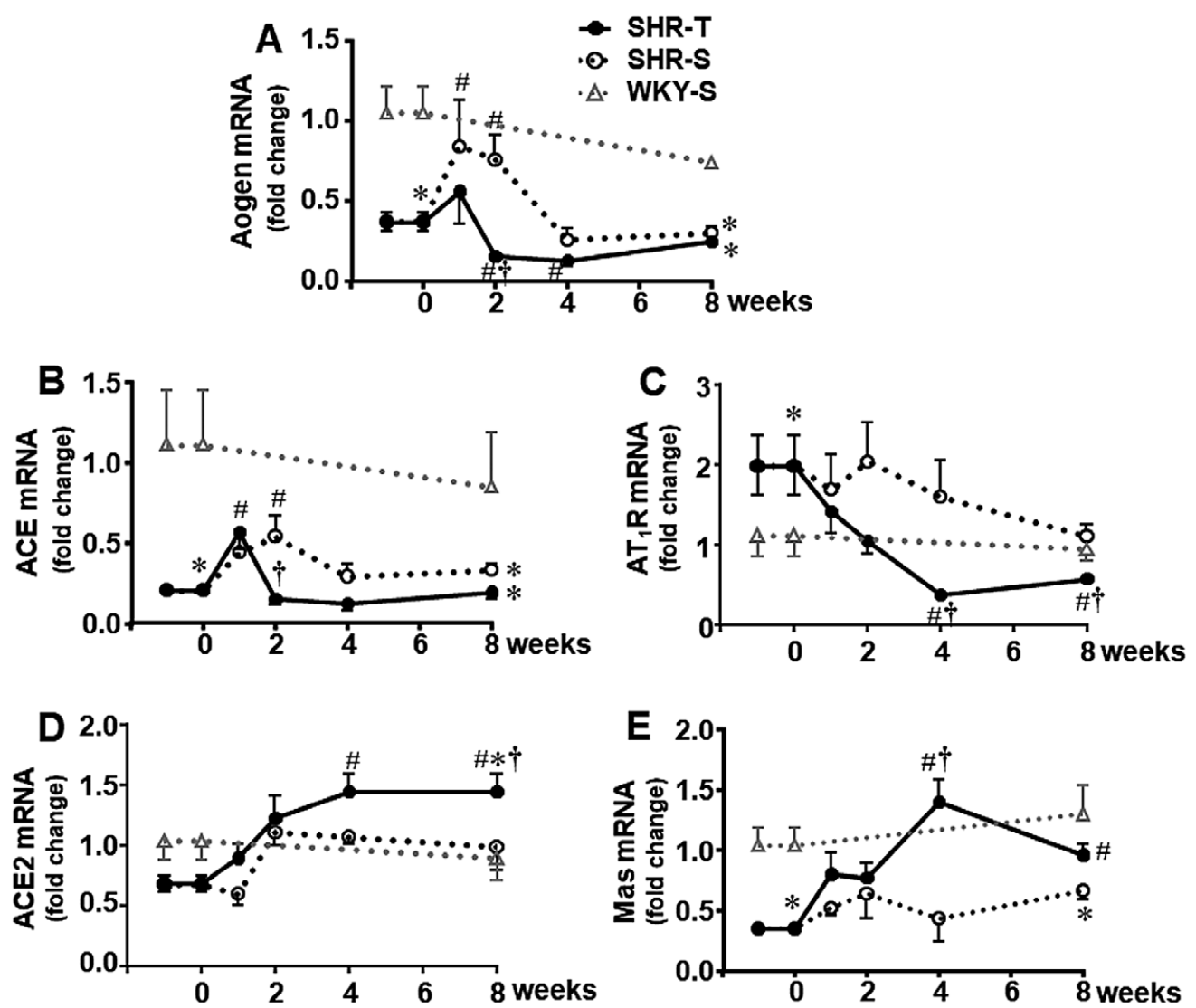

Figure 3. Hypertension- and training-induced changes on left ventricle renin-angiotensin system (RAS) gene expression during the development of hypertension in spontaneously hypertensive rats (SHR). Sequential changes on angiotensinogen (Aogen, A), angiotensin-converting enzyme (ACE, B), $A T_{1}$ receptor $\left(A T_{1} R, \mathbf{C}\right)$, angiotensin-converting enzyme 2 (ACE2, D), Mas receptor (MasR, E) gene expression in SHR and normotensive controls (Wistar Kyoto (WKY)). Measurements were made in 5-6 rats/time point/group. Significance $(P<0.05)$ : \#vs. respective week 0 ; * $v s$. age-matched WKY; †Vs. age-matched SHR-sedentary (S).

ings and determination of AP and HR and their variabilities at rest.,24 Rats were then deeply anesthetized for heart removal immediately after the respiratory arrest. LV hypertrophy was determined, and LV tissue was used for RAS gene (qPCR ${ }^{3,13}$; Table) and protein expression (Western Blotting $\left.{ }^{\mathbf{1 3}, 18}\right)$, histological and morphometric ${ }^{\mathbf{1 3}, 18}$ analyses. Myocytes diameter (hematoxylin-eosin-stained serial $10 \mu \mathrm{m}$ LV slices) and collagen content (picrosirius red-stained serial $5 \mu \mathrm{m}$ LV slices) were evaluated in a bright field Zeiss microscope by blind observers. Two-way factorial ANOVA followed by Tukey's HSD post-hoc test was used to compare groups and conditions.

An expanded Methods section is available in the Supplementary File.

\section{Results}

\section{Effects of Training on Treadmill Performance and Body Weight Gain}

Since the beginning of experiments, SHR showed better treadmill performance than age-matched WKY, as evidenced by the higher speed reached during maximal exercise tests $(1.67 \pm 0.05$ vs. $0.63 \pm 0.05 \mathrm{~km} / \mathrm{h}$, respectively, $\mathrm{P}<0.001$, Figure 1A). Aerobic training increased the veloc- ity attained by SHR-T $\left(+52 \%\right.$ and $+96 \%$ at the $4^{\text {th }}$ and $8^{\text {th }}$ experimental weeks, respectively, $\mathrm{P}<0.001)$. In contrast, SHR-S and WKY-S did not exhibit significant alterations in aerobic capacity (Figure 1A). Four-week-old SHR and WKY $(63 \pm 2 \mathrm{~g}$ and $67 \pm 3 \mathrm{~g})$ exhibited a similar weight gain $(+173 \pm 6 \mathrm{~g}$ and $+167 \pm 5 \mathrm{~g}$, respectively) during the 8 experimental weeks, with no difference between SHR-S and SHR-T.

\section{Sequential Effects of Hypertension and Training on Arterial Pressure and Pressure Variability}

At the beginning of the protocols, 4-week-old SHR exhibited similar MAP levels as age-matched WKY (110 \pm 8 and $104 \pm 5 \mathrm{mmHg}$, respectively, $\mathrm{P}=0.47$; Figure 1B). In $\mathrm{SHR}-\mathrm{S}$, there was a progressive MAP increase from the first (+15\%) up to eighth $(+56 \%)$ experimental week (Figure 1B). T did not abrogate the development of hypertension, but exhibited a small decrease in the first week, delayed the onset (significant increase only at the fourth experimental week) and reduced its magnitude (at the end of protocols SHR$\mathrm{T}=158 \pm 3 \mathrm{mmHg}$, SHR $-\mathrm{S}=172 \pm 3 \mathrm{mmHg}$, Figure 1B). Spectral analysis confirmed the efficacy of $\mathrm{T}$ in delaying and reducing the hypertensive effects. While SHR-S exhibited a progressive LF-SAP increase (significant from the fourth 

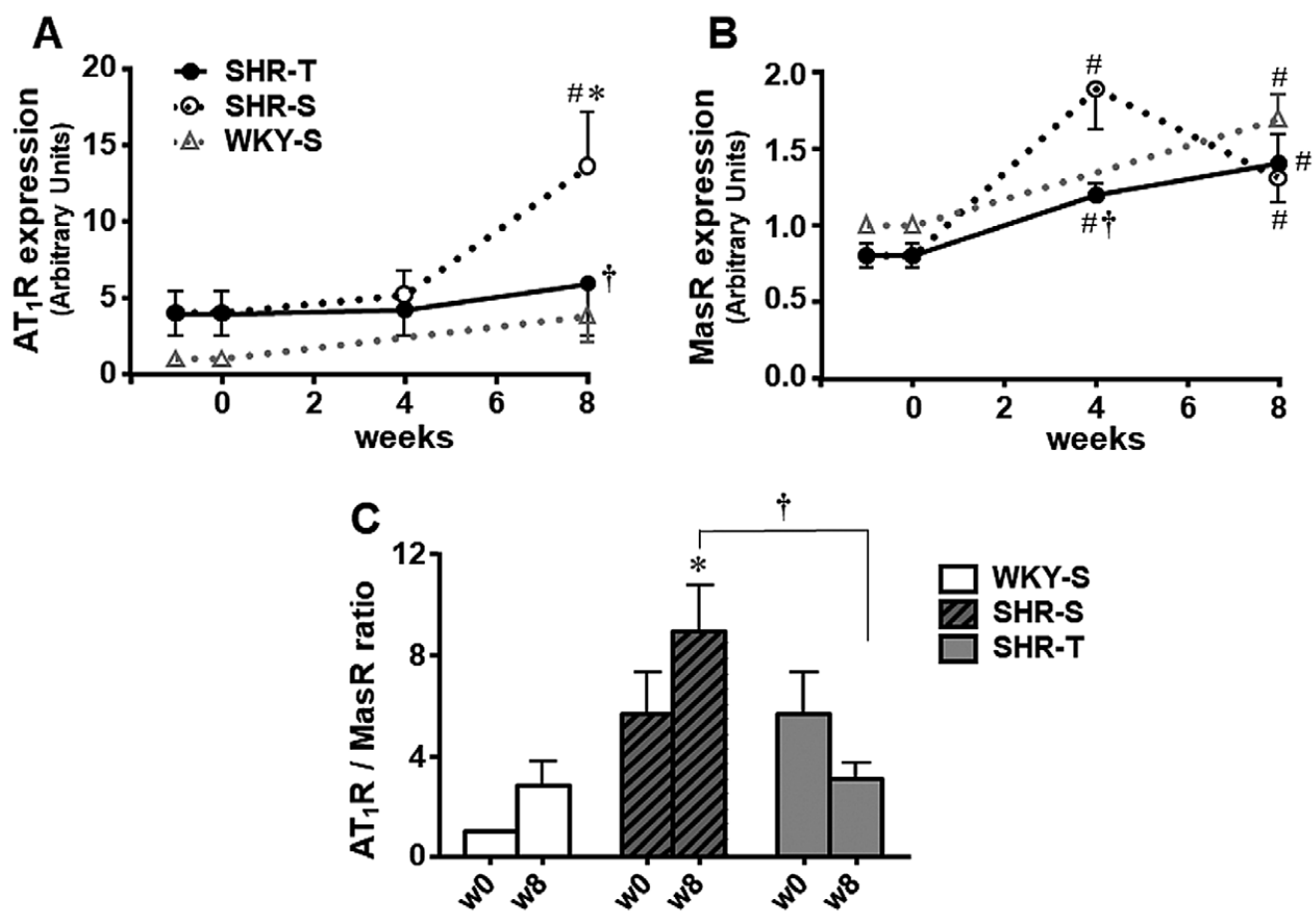

Figure 4. Hypertension- and training-induced changes on left ventricle protein expression of renin-angiotensin system (RAS) receptors during the development of hypertension in spontaneously hypertensive rats (SHR). Sequential changes on AT 1 receptor $\left(A T_{1} R, \mathbf{A}\right)$ and Mas receptor (MasR, B) expression in SHR and normotensive controls (Wistar Kyoto (WKY)) and changes on AT ${ }_{1}$ R/ MasR ratio at weeks 0 and 8 of experimental protocols. Measurements were made in $4-5$ rats/time point/group. Significance $(\mathrm{P}<0.05)$ : \#vs. respective week 0; *vs. age-matched WKY; †vs. age-matched SHR-sedentary $(\mathrm{S})$.

up to the eighth experimental week), T markedly delayed and reduced the sympathetic vasomotor activity in SHR-T (Figure 1D); SAP variability and hormonal modulation (VLF-SAP) were also significantly reduced in SHR-T when compared to age-matched SHR-S (Figure 1C and 1E, respectively).

\section{Sequential Effects of Hypertension and Training on HR, Pulse Interval (PI) Variability and Spontaneous Baroreflex Function Juvenile rats exhibited, at the beginning of protocols, high resting HR that was reduced with advancing age in all groups. Four-week-old SHR showed higher values than age-matched WKY $(437 \pm 31$ and $371 \pm 8$ beats/min respec- tively, $\mathrm{P}<0.05$, Figure 2A). HR falls were observed in both SHR groups since the fourth experimental week, but HR reduction was greater in SHR-T from the fourth week on, when values attained the same range exhibited by WKY-S (SHR-T =331 $\pm 8, \mathrm{WKY}-\mathrm{S}=322 \pm 7$ beats $/ \mathrm{min}$ ). The higher resting $\mathrm{HR}$ in juvenile SHR was accompanied by smaller PI variability $(-42 \%$, Figure $2 B)$ mainly due to reduced HF-PI (-59\% vs. age-matched WKY, Figure 2D). Sponta- neous baroreflex sensitivity was already depressed in juve- nile SHR-S (-50\% vs. WKY-S, Figure 2F). During the experimental protocol SHR-S showed significant increases in $\mathrm{LF}$ and $\mathrm{LF} / \mathrm{HF}$ ratio that were abrogated by training (Figure 2C,2E). Exercise training also augmented and almost normalized HF-PI from the fourth to eighth exper- imental week (Figure 2D), thus progressively increasing PI variability to a similar level exhibited by WKY at the eighth}

experimental week (Figure 2B). Spontaneous baroreflex sensitivity showed an increasing trend in the SHR-T when compared to SHR-S group (Figure 2F).

\section{Sequential Effects of Hypertension and Training on LV RAS Gene and Protein Expression}

Knowing that cardiac RAS is a key regulator of LV remodeling in hypertensive individuals, ${ }^{11,13,16,22}$ we investigated the sequential effects of hypertension and exercise on gene and protein expression of vasoconstrictor and vasodilator RAS axes during the establishment of hypertension. Compared to age-matched WKY-S, pre-hypertensive SHR exhibited smaller angiotensinogen (Aogen), ACE and MasR (-65\%, $-82 \%$ and $-66 \%$, respectively, $\mathrm{P}<0.05$, Figure 3A,3B,3E), but a higher LV AT 1 R mRNA level (+78\%, P >0.05, Figure 3C). While mRNA expression of precursor and other RAS components did not change in WKY-S during the experimental weeks, Aogen and ACE mRNA of the SHR-S showed significant increases at the first and second experimental weeks (+2.3- and +2.7-fold vs. basal levels, corresponding to 5 and 6 weeks of age, Figure $\mathbf{3 A}$ and $\mathbf{3 B}$, respectively) when MAP started raising. These changes were not maintained throughout the fourth to eighth experimental weeks. In the SHR-S, AT 1 R, ACE2 and MasR mRNA levels were not significantly changed during the 8 experimental weeks (Figure 3C-3E). Importantly, aerobic training mitigated and shortened the transient increase in Aogen mRNA expression exhibited by SHR-S (Figure 3A) and altered the time course changes of both RAS axes during the transition 


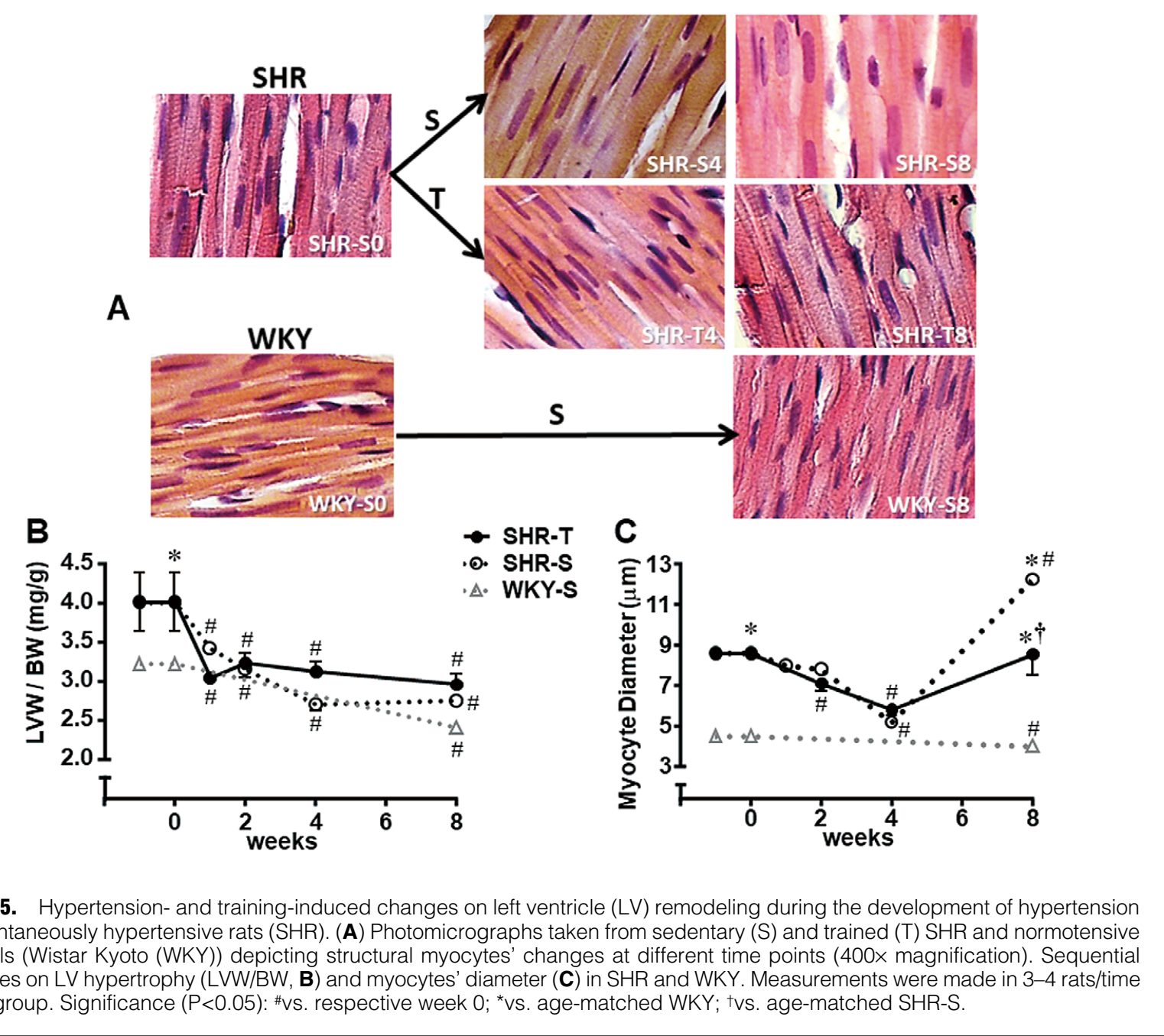

from the pre-hypertensive to hypertensive phase. There was a transient ACE increase in SHR-T during the first experimental week (corresponding to 5 weeks of age) that was abrogated by training from the second week on (Figure 3B). The marked T-induced decrease in $\mathrm{AT}_{1} \mathrm{R}$ gene expression, significant since the fourth experimental week $(-80 \%$ from basal levels, Figure 3C), also contributed to the downregulation of the vasoconstrictor-trophic-oxidative-inflammatory RAS axis. At the end of the protocols, SHR-T AT 1 R mRNA expression was lower than that for age-matched SHR-S. In addition, the contraregulatory axis was simultaneously augmented in the LV of the SHR-T; there was a marked increase in ACE2 mRNA expression from 4 up to 8 weeks training ( 2 -fold increase, Figure 3D) accompanied by increased MasR mRNA expression that peaked at the fourth experimental week (4-fold increase, Figure 3E) and was still 2.7-fold higher than basal levels at the end of protocols.

Gene expression changes were confirmed by sequential analyzes of $\mathrm{LV} \mathrm{AT} 1 \mathrm{R}$ and MasR protein expression. In arbitrary normalized units, $\mathrm{AT}_{1} \mathrm{R}$ of pre-hypertensive SHR was 5-fold higher than MasR values (3.99 \pm 1.45 vs. $0.80 \pm 0.08$, Figure 4A,4B). At the beginning of protocols, there was a trend for higher $\mathrm{AT}_{1} \mathrm{R}$ and smaller MasR in SHR-S vs.
WKY-S (Figure 4A,4B, P>0.05). AT 1 R expression was not changed in WKY-S; in contrast, it was largely increased in SHR-S at the eighth experimental week (+3.4-fold, Figure 4A). This increase was completely blocked in SHR$\mathrm{T}$, whose value was like that of WKY-S. During the experimental protocols, MasR protein expression increased in all groups $(\sim 1.7$-fold at the eighth week), with a large transient compensatory increment in the SHR-S at the fourth week $(+2.4$-fold, Figure 4B). Training-induced changes in SHR receptors' expression during the transition from the pre-hypertensive to hypertensive phase were more pronounced in the vasoconstrictor (marked $A T_{1} R$ reduction at the eighth week) than in the vasodilator axis (mild MasR increase at the fourth experimental week). As depicted in Figure 4C, the high arbitrary normalized values of $A T_{1} R$ relative to respective MasR yielded positive $A T_{1} R /$ MasR ratios during the 8 experimental weeks. SHR-S $\mathrm{AT}_{1} \mathrm{R} / \mathrm{MasR}$ ratio was slightly but not significantly higher than WKY-S at the pre-hypertensive phase (week 0), but largely increased at the eighth experimental week (rats aged 3 months). Interestingly, 8 weeks of aerobic training did not block the establishment of hypertension (although it did reduce it, Figure 1B), but it was largely effective to reduce the $\mathrm{AT}_{1} \mathrm{R} / \mathrm{MasR}$ ratio back to normotensive levels. 


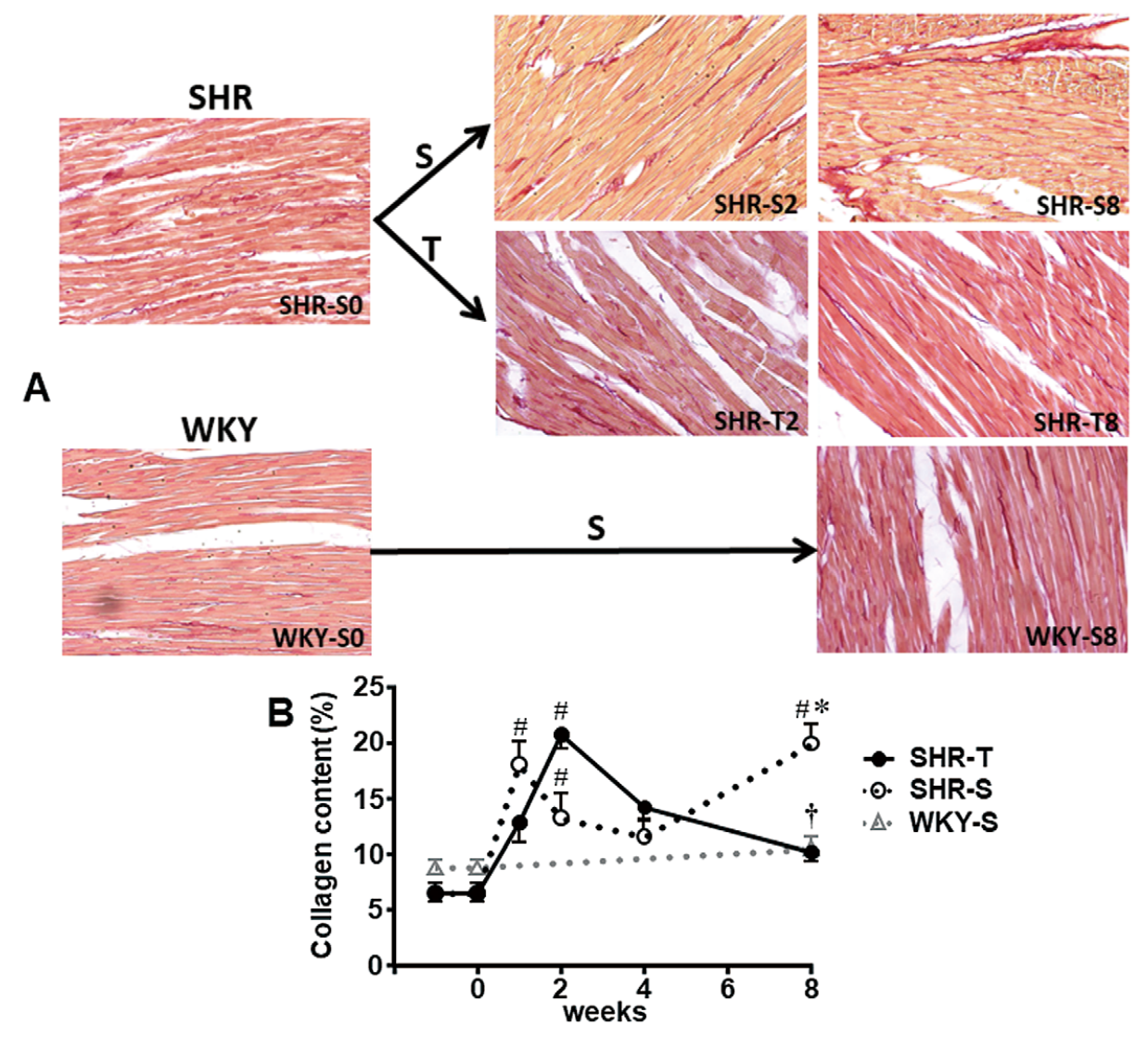

Figure 6. Hypertension- and training-induced changes on left ventricle (LV) collagen deposition during the development of hypertension in spontaneously hypertensive rats (SHR). (A) Photomicrographs taken from sedentary (S) and trained (T) SHR and normotensive controls (Wistar Kyoto (WKY)) depicting myocytes' collagen content at different time points. (200x magnification). Sequential changes on LV collagen deposition (B) in SHR and WKY. Measurements were made in 3-4 rats/time point/group. Significance $(\mathrm{P}<0.05)$ : \#vs. respective week 0 ; *vs. age-matched WKY; †vs. age-matched SHR-S.

\section{Sequential Changes on Cardiac Remodeling}

RAS hyperactivity and LV hypertrophy characterized the chronic hypertension. ${ }^{11,13,22,25}$ To uncover the time-course changes in LV remodeling during the establishment of hypertension and the effects of exercise, we measured the LVW/BW ratio and the sequential changes in LV myocytes' diameter. Pre-hypertensive SHR already exhibited

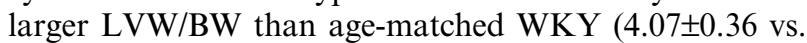
$3.22 \pm 0.07 \mathrm{mg} / \mathrm{g}$, Figure 5B). LVW/BW ratio was reduced in all groups during the 8 experimental weeks, without significant differences between them. At the beginning of protocols, LV myocytes' diameter was also higher in SHR vs. WKY $(8.6 \pm 0.2 \mu \mathrm{m}$ vs. $4.5 \pm 0.1 \mu \mathrm{m}$, respectively, Figure 5A and 5C). Similar to LVW/BW, myocytes' diameter of both SHR-S and SHR-T decreased from the first to fourth experimental week, but the establishment of hypertension reversed this effect: SHR-S diameters' size started to increase markedly from the fourth week on, while SHR-T only showed a small increase. At the end of protocols, SHR-T exhibited smaller myocytes' diameter than SHR-S (8.5 \pm 1.0 vs. $12.2 \pm 0.1 \mu \mathrm{m}$, respectively, Figure 5C).

Knowing that collagen accumulation contributes to cardiac remodeling in hypertension, ${ }^{\mathbf{1 0 , 1 3 , 1 4 , 2 6}}$ we also analyzed the temporal changes of collagen content during the transi- tion from the pre- to hypertensive phase. There was no difference in LV collagen content between juvenile SHR and age-matched controls (Figure 6A and 6B). While collagen content was not changed in WKY-S, the establishment of hypertension augmented LV collagen deposition that was more precocious and intense in SHR-S and milder in the SHR-T, specifically at the end of protocols when training was effective in reducing $\mathrm{AT}_{1} \mathrm{R} / \mathrm{MasR}$ ratio (compare Figures 6B and 4C).

\section{Discussion}

The present set of data confirmed the involvement of the vasoconstrictor-trophic-pro-oxidative-pro-inflammatory RAS axis in SHR LV hypertrophy and that pre-hypertensive exercise training attenuates both hypertension and cardiac remodeling. In addition, it identified the sequential mechanisms that drive the hemodynamic and autonomic control and the $\mathrm{LV}$ remodeling during the transition from the pre-hypertensive to hypertensive phase. Our findings are as follow: (1) at the pre-hypertensive phase, young SHR exhibits lower Aogen, ACE and MasR expression, but an already increased AT 1 R mRNA expression; (2) the development of hypertension starts earlier in SHR-S (at 


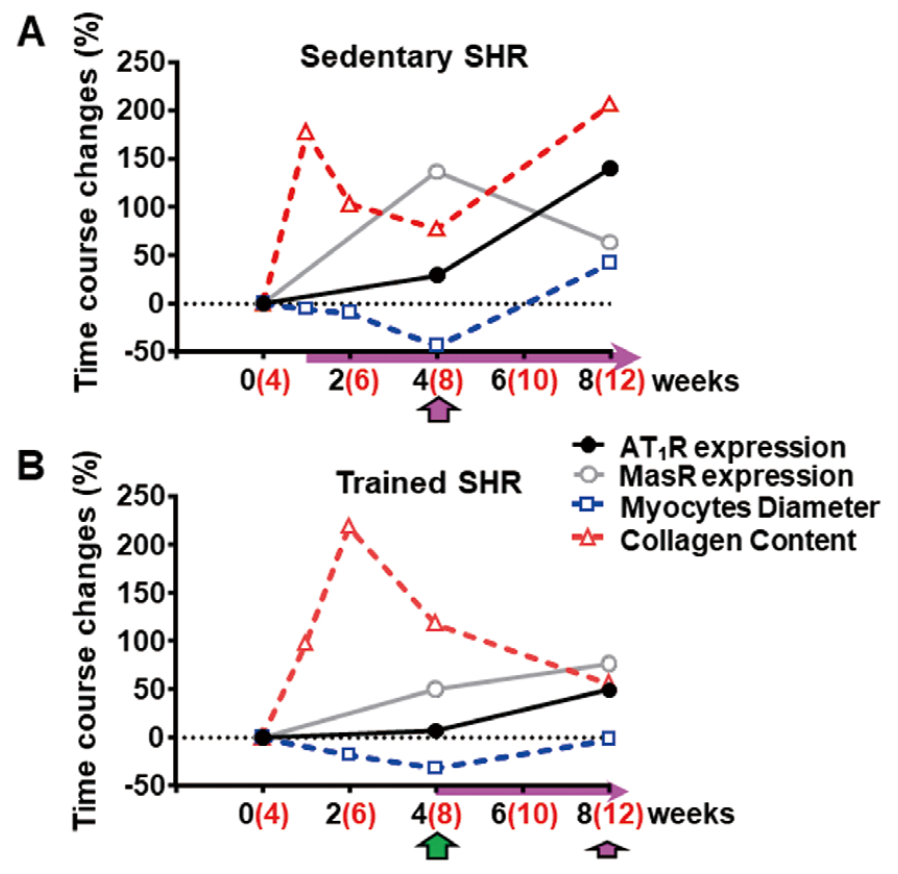

\begin{abstract}
Figure 7. Schematic representation of temporal changes of $A T_{1}$ and Mas receptors expression and left ventricle remodeling in sedentary $(\mathbf{A})$ and trained (B) spontaneously hypertensive rats (SHR) during the transition from the pre- to hypertensive phase. The black numbers on the $\mathrm{X}$-axis indicate the experimental weeks and red numbers in parenthesis represent the age of rats at that time point. The longitudinal pink arrows represent the incidence of hypertension; the arrows pointing upwards indicate the time point in which sympathetic (pink) and parasympathetic activity (green) starts to increase. The relative width and size of arrows are indicative of the magnitude of changes.
\end{abstract}

approximately the fifth week of age) and occurs simultaneously with increased LV Aogen and ACE expression, which is suggestive of high Ang II availability, and augmented LV collagen deposition; (3) the pressure increase in SHR-S is accompanied by reduced spontaneous baroreflex sensitivity, progressive augmentation in the sympathetic modulation of heart/vessels since the fourth experimental week ( 8 weeks old), and by increased $\mathrm{AT}_{1} \mathrm{R}$ expression, augmented pressure variability and deleterious LV remodeling at the end of protocols (12 weeks old); and (4) exercise training abrogates the initial hypertension-induced increase in Aogen and ACE expression and the late augmentation in $\mathrm{AT}_{1} \mathrm{R}$, improves the expression of the contraregulatory RAS axis, decreases the sympathetic and increases the parasympathetic modulation of the heart, blocks LV myocytes' hypertrophy and collagen deposition, while delaying and reducing vasomotor sympathetic activity, pressure levels and pressure variability. As expected, exercise training also increases PI variability and reduces resting HR, two important markers of trainability.

The pivotal role of RAS in the maintenance of hypertension, autonomic dysfunction and cardiac hypertrophy in the SHR is well known. ${ }^{27}$ Increased plasma and tissue RAS expression, high Ang II availability, sympathetic hyperactivity, oxidative stress and pro-inflammatory profile, myocytes' hypertrophy and increased LV collagen synthesis were observed in the SHR. 3,13,18,26,28-30 Pharmacological studies showed that ACE inhibitors, $\mathrm{AT}_{1} \mathrm{R}$ receptor antagonists and RAS silencing attenuated hypertension and heart hypertrophy, ${ }^{31}$ and that $\mathrm{AT}_{1} \mathrm{R}$ antisense gene therapy reduced cardiac hypertrophy independently of pressure changes. ${ }^{25}$ Blockade of the $\mathrm{AT}_{1} \mathrm{R}$ also reduced Ang II intracellular signaling, oxidative stress, myocytes' hypertrophy, LV fibrosis and collagen deposition in the SHR. ${ }^{32,33}$

As those studies were conducted in adult/aged and already hypertensive SHR, we focused our observations in the transition from the pre- to hypertensive phase. Our data indicated an already increased $\mathrm{AT}_{1} \mathrm{R}$ mRNA expression in young pre-hypertensive SHR-S. The augmented gene expression of both Aogen and the ACE-Ang II-AT 1 R RAS axis at the onset of hypertension are decisive to trigger the progressive increase in pressure (since the fifth week of age, Figure 7A) and sympathoexcitation (increased further in SHR-S aged 8 weeks). Moreover, a deleterious cardiac remodeling and a marked augmentation in $\mathrm{LV} \mathrm{AT}_{1} \mathrm{R}$ protein content were observed when SHR were 12 weeks old (Figure 7A). The transient compensatory increase in the contraregulatory axis was not enough to block the increased activity of the vasoconstrictor-trophic-pro-oxidative-proinflammatory RAS axis, which worsened LV function and cardiac fibrosis. The essential role played by the vasoconstrictor axis was previously confirmed by the blockade of these effects following $\mathrm{AT}_{1} \mathrm{R}$ antagonism. ${ }^{\mathbf{3 4 - 3 6}}$ It was also shown that collagen content changes occurred independently of pressure changes. ${ }^{35}$ The early increase in the ACE-Ang II-AT $1 \mathrm{R}$ axis could also contribute to augment the sympathetic activity to the heart, $\mathbf{5 , 3 7 , 3 8}$ which is an additional stimulus to potentiate the late myocyte hypertrophy and collagen deposition. Indeed, the elevated resting HR in pre-hypertensive SHR indicated an already high cardiac sympathetic activity, which was further increased from the eighth week of age on (Figure 7A).

Interestingly, myocytes' diameter of pre-hypertensive SHR was almost 2-fold higher than that of age-matched normotensive controls. Keeping with the known developmental tendency of the biological aging progress, ${ }^{37}$ myocytes' diameter and LVW/BW ratio, as well as resting HR, decreased with age (Figure 2A and Figure 5B,5C). However, the SHR-S myocytes' diameter reduction is interrupted and reversed by the development of hypertension, attaining a high value in the chronic phase. At this time point, pressure variability, $\mathrm{AT}_{1} \mathrm{R}$ expression and $\mathrm{LV}$ collagen content were 
also largely augmented (Figure 7A). As shown by Kudo et al, ${ }^{39}$ exaggerated pressure variability superimposed on hypertension aggravated the deleterious cardiac remodeling via Ang II-mediated chronic inflammation.

Figure 7B depicts an important finding of this study. Aerobic training did not interfere with the development of genetic hypertension, but delayed the onset by 3 weeks, reduced its magnitude and postponed the increase in vasomotor sympathetic activity by 4 weeks. Importantly, it offset the hypertension-induced rise in cardiac $A T_{1} R$ expression and facilitated MasR expression from the fourth experimental week on. These training-adaptive changes increased parasympathetic modulation of the heart (fourth experimental week), decreased and postponed hypertension-related sympathoexcitation (Figure 7B), thus normalizing HR variability and reducing pressure variability, essential mechanisms to reduce end-organ damage. Indeed, 8 -weeks of repetitive exercise was enough to normalize LV collagen deposition and to markedly reduce myocytes' diameter (Figure 7B). It is well known that exercise training is an efficient tool to treat hypertension. ${ }^{40}$ Experimental data successively reported that 8 weeks of exercise training normalized both autonomic control and baroreflex sensitivity and reduced pressure in adult SHR by downregulating tissue RAS and decreasing Ang II availability, both central and peripherally, $3,13,18,30$ therefore reducing the activation of intracellular signaling pathways leading to oxidative and pro-inflammatory profiles. ${ }^{3,4}$ In young SHR-T, it was reported that chronic exercise attenuated tail-pressure raise, reduced cardiac Ang II and increased Ang-(1-7) content, restored the balance between pro- and anti-inflammatory cytokines, attenuated both cardiac hypertrophy and fibrosis, and restored LV function. ${ }^{22,41}$ Loss-of-function studies also confirm that Ang-(1-7) has a protective role in the development of cardiac remodeling in different hypertension models. ${ }^{42,43}$ The LV and plasma Ang II and Ang(1-7) measurements in exercised adult SHR indicated that training-induced Ang II reduction was a fast response (first to second experimental week), with LV changes preceding that of plasma by 1 week. ${ }^{13}$ In contrast, exercise-induced augmentation in Ang-(1-7) content occurred first in the plasma and later in the LV (second week on). ${ }^{13}$ These time course changes reinforce the temporal changes now observed; in the SHR-T, the reduction of LV ACE mRNA expression preceded that of ACE2 mRNA increase, indicating that the transition from the pre- to hypertensive phase was characterized by a precocious deactivation of the vasoconstrictor-trophic-pro-oxidative-pro-inflammatory axis followed by a late activation of the contraregulatory RAS axis. In support to our findings, Peng et a ${ }^{22}$ also showed that ACE-Ang II-AT 1 R and ACE2-Ang-(1-7)MasR axes exhibited different temporal responses, with the vasodilator-anti-trophic-anti-oxidative-anti-inflammatory RAS axis being a late adaptive change.

In this study, we only evaluated the temporal changes of LV RAS axes, but as demonstrated by Brilla and Keler, the elevated aldosterone levels induced cardiac hypertrophy and fibrosis, ${ }^{44}$ and it is likely that aldosterone and mineralocorticoid receptor (MR) must be involved. Indeed, Yasuoka et a ${ }^{45}$ have shown that cardiac MR activation in the SHR increased myocytes' diameter and aggravated both fibrosis and LV hypertrophy. It remains to be determined whether the transition from the pre- to hypertensive phase also courses with LV changes in aldosterone and MR.

The early transient increase in LV collagen content observed in the trained SHR at the second week was most likely due to the acute inflammatory effect at starting the exercise. As showed previously in adult SHR, opposite to regular exercise training, single bouts of exercise augment redox signaling and NF-kB translocation to the nucleus, thus increasing TNF $\alpha$ and IL- $1 \beta$ expression in the heart (as well as in the skeletal muscle). ${ }^{13}$ This effect, however, persisted only during the first 2 weeks of training, being replaced by a late increase in IL-10, ${ }^{13}$ which favored the marked reduction in collagen deposition observed at the end of the training protocol (Figure 7B).

\section{Conclusions}

Our data that analyzed the temporal effects of RAS axes in LV remodeling during the transition from the pre- to hypertensive phase in sedentary and trained SHR uncover the pivotal role played by aerobic training in protecting the heart against deleterious remodeling. Repetitive exercise is accompanied by early downregulation of the ACE-Ang II-AT 1 R axis followed by upregulation of the contraregulatory RAS axis, both contributing to improve the autonomic control and to avoid myocytes' hypertrophy and LV collagen deposition. These adaptive responses contribute to a delay and a decrease in the magnitude of hypertension, reducing the risk for end-organ damage, even in the presence of hypertension.

\section{Disclosures}

The authors declare no potential conflicts of interest concerning the research, authorship and/or publication of this article.

\section{Funding}

This study was supported by Fundação de Amparo à Pesquisa do Estado de São Paulo [FAPESP research grant 15/24935-4 to L.C.M.)], Conselho Nacional de Desenvolvimento Científico e Tecnológico [CNPq, fellowship to TSRC and grant 305940/2015-5 (research fellow to L.C.M.)] and Coordenação de Aperfeiçoamento de Pessoal de Nível Superior - Brazil [CAPES - Finance Code 001 and CAPES/ PNPD 1752953, fellowship to G.S.M.].

\section{References}

1. Folkow B. Physiological aspects of primary hypertension. Physiol Rev 1982; 62: 347-504.

2. Calvillo L, Gironacci MM, Crotti L, Meroni PL, Parati G. Neuroimmune crosstalk in the pathophysiology of hypertension. Nat Rev Cardiol 2019; 16: 476-490.

3. Masson GS, Costa TS, Yshii L, Fernandes DC, Soares PP, Laurindo FR, et al. Time-dependent effects of training on cardiovascular control in spontaneously hypertensive rats: Role for brain oxidative stress and inflammation and baroreflex sensitivity. PLoS One 2014; 9: e94927.

4. Buttler L, Jordão MT, Fragas MG, Ruggeri A, Ceroni A, Michelini LC. Maintenance of blood-brain barrier integrity in hypertension: A novel benefit of exercise training for autonomic control. Front Physiol 2017; 8: 1048.

5. Shanks J, Manou-Stathopoulou S, Lu CJ, Li D, Paterson DJ, Herring N. Cardiac sympathetic dysfunction in the prehypertensive spontaneously hypertensive rat. Am J Physiol Heart Circ Physiol 2013; 305: H980-H986.

6. Hirst A, Marshall JM. Endothelium-dependent and cyclooxygenase-dependent cutaneous vasodilatation is blunted in young men with hypertensive parents. J Hypertens 2018; 36: 2140-2147.

7. Naiel S, Carlisle RE, Lu C, Tat V, Dickhout JG. Endoplasmic reticulum stress inhibition blunts the development of essential hypertension in the spontaneously hypertensive rat. Am J Physiol Heart Circ Physiol 2019; 316: H1214-H1223.

8. Forrester SJ, Booz GW, Sigmund CD, Coffman TM, Kawai T, Rizzo V. Angiotensin II signal transduction: An update on mechanisms of physiology and pathophysiology. Physiol Rev 2018; 98: $1627-1738$ 
9. Santos RAS, Sampaio WO, Alzamora AC, Motta-Santos D, Alenina N, Bader M. The ACE2/Angiotensin-(1-7)/MAS axis of the renin-angiotensin system: Focus on angiotensin-(1-7). Physiol Rev 2018; 98: 505-553.

10. González A, Ravassa S, López B, Moreno MU, Beaumont J, San José G. Myocardial remodeling in hypertension. Hypertension 2018; 72: 549-558

11. Xu J, Carretero OA, Liao TD, Peng H, Shesely EG, Xu J. Local angiotensin II aggravates cardiac remodeling in hypertension. Am J Physiol Heart Circ Physiol 2010; 299: H1328-H1338.

12. $\mathrm{Xu} \mathrm{S}$, Zhi H, Hou X, Cohen RA, Jiang B. I $\kappa \mathrm{B} \beta$ attenuates angiotensin II-induced cardiovascular inflammation and fibrosis in mice. Hypertension 2011; 58: 310-316.

13. Silva SD Jr, Jara ZP, Peres R, Lima LS, Scavone C, Montezano $\mathrm{AC}$ et al. Temporal changes in cardiac oxidative stress, inflammation and remodeling induced by exercise in hypertension: Role for local angiotensin II reduction. PLoS One 2017; 12: e0189535.

14. Tsutsui H, Matsushima S, Kinugawa S, Ide T, Inoue N, Ohta Y et al. Angiotensin II type 1 receptor blocker attenuates myocardial remodeling and preserves diastolic function in diabetic heart. Hypertens Res 2007; 30: 439-449.

15. Gomes ER, Lara AA, Almeida PW, Guimarães D, Resende RR, Campagnole-Santos MJ, et al. Angiotensin-(1-7) prevents cardiomyocyte pathological remodeling through a nitric oxide/guanosine 3',5'-cyclic monophosphate-dependent pathway. Hypertension 2010; 55: $153-160$

16. Santiago NM, Guimarães PS, Sirvente RA, Oliveira LA, Irigoyen MC, Santos RA, et al. Lifetime overproduction of circulating Angiotensin-(1-7) attenuates deoxycorticosterone acetate-salt hypertension-induced cardiac dysfunction and remodeling. Hypertension 2010; 55: 889-896.

17. Zamo FS, Barauna VG, Chiavegatto S, Irigoyen MC, Oliveira EM. The renin-angiotensin system is modulated by swimming training depending on the age of spontaneously hypertensive rats. Life Sci 2011;89: 93-99.

18. Silva SD Jr, Zampieri TT, Ruggeri A, Ceroni A, Aragão DS, Fernandes FB, et al. Downregulation of the vascular renin-angiotensin system by aerobic training: Focus on the balance between vasoconstrictor and vasodilator axes. Circ J 2015; 79: 1372-1380.

19. Gomes-Filho A, Ferreira AJ, Santos SHS, Neves SRS, Silva Camargos ER, Becker LK, et al. Selective increase of angioten$\sin (1-7)$ and its receptor in hearts of spontaneously hypertensive rats subjected to physical training. Exp Physiol 2008; 93: 589-598

20. Fernandes T, Hashimoto NY, Magalhães FC, Fernandes FB Casarini DE, Carmona AK, et al. Aerobic exercise training-induced left ventricular hypertrophy involves regulatory MicroRNAs, decreased angiotensin-converting enzyme-angiotensin II, and synergistic regulation of angiotensin-converting enzyme 2-angiotensin (1-7). Hypertension 2011; 58: 182-189.

21. Nunes-Silva A, Rocha GC, Magalhaes DM, Vaz LN, Salviano de Faria MH, Simoes E Silva AC. Physical exercise and ACE2angiotensin-(1-7)-Mas receptor axis of the renin angiotensin system. Protein Pept Lett 2017; 24: 809-816.

22. Peng WW, Hong L, Liu GY, Lin C, Zhao XL, Wang SZ, et al. Prehypertension exercise training attenuates hypertension and cardiac hypertrophy accompanied by temporal changes in the levels of angiotensin II and angiotensin (1-7). Hypertens Res 2019; 42: $1745-1756$.

23. Masson GS, Nair AR, Silva Soares PP, Michelini LC, Francis J. Aerobic training normalizes autonomic dysfunction, HMGB1 content, microglia activation and inflammation in hypothalamic paraventricular nucleus of SHR. Am J Physiol Heart Circ Physiol 2015; 309: H1115-H1122

24. Ceroni A, Chaar LJ, Bombein RL, Michelini LC. Chronic absence of baroreceptor inputs prevents training-induced cardiovascular adjustments in normotensive and spontaneously hypertensive rats. Exp Physiol 2009; 94: 630-640.

25. Pachori AS, Numan MT, Ferrario CM, Diz DM, Raizada MK, Katovich MJ. Blood pressure-independent attenuation of cardiac hypertrophy by AT(1)R-AS gene therapy. Hypertension 2002; 39: 969-975.

26. Sano H, Okamoto H, Kitabatake A, Iizuka K, Murakami T, Kawaguchi H. Increased mRNA expression of cardiac reninangiotensin system and collagen synthesis in spontaneously hypertensive rats. Mol Cell Biochem 1998; 178: 51-58.

27. Trippodo NC, Frohlich ED. Similarities of genetic (spontaneous) hypertension: Man and rat. Circ Res 1981; 48: 309-319.
28. Gray MO, Long CS, Kalinyak JE, Li HT, Karliner JS. Angiotensin II stimulates cardiac myocyte hypertrophy via paracrine release of TGF-beta 1 and endothelin- 1 from fibroblasts. Cardiovasc Res 1998; 40: 352-363.

29. Schultz JJ, Witt SA, Glascock BJ, Nieman ML, Reiser PJ, Nix SL et al. TGF-betal mediates the hypertrophic cardiomyocyte growth induced by angiotensin II. J Clin Invest 2002; 109: 787-796.

30. Chaar LJ, Alves TP, Batista Junior AM, Michelini LC. Early training-induced reduction of angiotensinogen in autonomic areas: The main effect of exercise on brain renin-angiotensin system in hypertensive rats. PLoS One 2015; 10: e0137395.

31. Kimura B, Mohuczy D, Tang X, Phillips MI. Attenuation of hypertension and heart hypertrophy by adeno-associated virus delivering angiotensinogen antisense. Hypertension 2001; 37: 376-380.

32. Makino N, Sugano M, Otsuka S, Hata T. Molecular mechanism of angiotensin II type I and type II receptors in cardiac hypertrophy of spontaneously hypertensive rats. Hypertension 1997; 30: $796-802$.

33. Ito N, Ohishi M, Yamamoto K, Tatara Y, Shiota A, Hayashi N, et al. Renin-angiotensin inhibition reverses advanced cardiac remodeling in aging spontaneously hypertensive rats. $\mathrm{Am} \mathrm{J}$ Hypertens 2007; 20: $792-799$.

34. Baumann M, Hermans JJ, Janssen BJ, Peutz-Kootstra C, Witzke $\mathrm{O}$, Heemann U, et al. Transient prehypertensive treatment in spontaneously hypertensive rats: A comparison of spironolactone and losartan regarding long-term blood pressure and target organ damage. J Hypertens 2007; 25: 2504-2511.

35. Varagic J, Frohlich ED, Susic D, Ahn J, Matavelli L, López B, et al. AT1 receptor antagonism attenuates target organ effects of salt excess in SHRs without affecting pressure. Am J Physiol Heart Circ Physiol 2008; 294: H853-H858.

36. He DH, Zhang LM, Lin LM, Ning RB, Wang HJ, Xu CS, et al. Effects of losartan and amlodipine on left ventricular remodeling and function in young stroke-prone spontaneously hypertensive rats. Acta Cardiol Sin 2014; 30: 316-324.

37. Dang A, Zheng D, Wang B, Zhang Y, Zhang P, Xu M, et al. The role of the renin-angiotensin and cardiac sympathetic nervous systems in the development of hypertension and left ventricular hypertrophy in spontaneously hypertensive rats. Hypertens Res 1999; 22: 217-221.

38. Levick SP, Murray DB, Janicki JS, Brower GL. Sympathetic nervous system modulation of inflammation and remodeling in the hypertensive heart. Hypertension 2010; 55: 270-276.

39. Kudo H, Kai H, Kajimoto H, Koga M, Takayama N, Mori T, et al. Exaggerated blood pressure variability superimposed on hypertension aggravates cardiac remodeling in rats via angiotensin II system-mediated chronic inflammation. Hypertension 2009; 54: $832-838$.

40. Cornelissen VA, Smart NA. Exercise training for blood pressure: A systematic review and meta-analysis. J Am Heart Assoc 2013; 2: $\mathrm{e} 004473$.

41. Jia LL, Kang YM, Wang FX, Li HB, Zhang Y, Yu XJ, et al. Exercise training attenuates hypertension and cardiac hypertrophy by modulating neurotransmitters and cytokines in hypothalamic paraventricular nucleus. PLoS One 2014; 9: e85481.

42. Yamamoto K, Ohishi M, Katsuya T, Ito N, Ikushima M, Kaibe $\mathrm{M}$, et al. Deletion of angiotensin-converting enzyme 2 accelerates pressure overload-induced cardiac dysfunction by increasing local angiotensin II. Hypertension 2006; 47: 718-726.

43. Zhong J, Basu R, Guo D, Chow FL, Byrns S, Schuster M, et al. Angiotensin-converting enzyme 2 suppresses pathological hypertrophy, myocardial fibrosis, and cardiac dysfunction. Circulation 2010; 122: 717-728.

44. Kritis AA, Gouta CP, Liaretidou EI, Kallaras KI. Latest aspects of aldosterone actions on the heart muscle. J Physiol Pharmacol 2016; 67: $21-30$.

45. Yasuoka S, Hisashi K, Kajimoto H, Kudo H, Takayama N, Anegawa T, et al. Blood pressure variability activates mineralocorticoid receptor and induces cardiac remodeling in hypertensive rats. Circ J 2013; 77: 1474-1481.

\section{Supplementary Files}

Please find supplementary file(s);

http://dx.doi.org/10.1253/circj.CJ-19-1161 